\title{
LVIII. On the rotation of a rigid body about a fixed point
}

\section{J.J. Sylvester M.A. F.R.S.}

To cite this article: J.J. Sylvester M.A. F.R.S. (1850) LVIII. On the rotation of a rigid body about a fixed point, Philosophical Magazine Series 3, 37:252, 440-444, DOI: $10.1080 / 14786445008646649$

To link to this article: http://dx.doi.org/10.1080/14786445008646649

曲 Published online: 30 Apr 2009.

Submit your article to this journal $₫$

Џll Article views: 2

Q View related articles $\asymp$ 
LVIII. On the Rotation of a Rigid Body about a Fixed Point. By J. J. Sylvester, M.A., F.R.S.*

TN the Cambridge and Dublin Mathematical Journal for 1 March 1848, an article by Professor Stokes, of the University of Cambridge, is ushered in with the words following:"The most general instantaneous + motion of a rigid body moveable in all directions about a fixed point consists in a motion of rotation about an axis passing through that point. This elementary proposition is sometimes assumed as selfevident, and sometimes deduced as the result of an analytical process. It ought hardly perhaps to be assumed, but it does not seem desirable to refer to a long algebraical process for the demonstration of a theorem so simple. Yet I am not aware of a geometrical proof anywhere published which might be referred to."

The learned and ingenious professor is indubitably right, and might have trusted himself to assert less hesitatingly the necessity of demonstrating this proposition, which possesses none of the characters of a self-evident truth; but it is to be regretted that he should have stated it in such a form as naturally to lead the incautious reader to mistake the nature and grounds of its existence, which consist in this fact-that any kind of displacement of a body moveable about a fixed axis, whether instantaneous and infinitesimal, or secular and finite, is capable of being effected by a single rotation about a single axis.

The annexed simple proof of this capital law has the advantage of affording a rule for compounding into one any two (and therefore any number of) rotations given in direction, magnitude and order of succession.

It will somewhat conduce to simplicity if we fix our attention upon a spherical surface rigidly connected with the rotating body, and having its centre at the fixed point thereof. When the positions of two points in this are given, the position of the body is completely determined.

Now evidently two points $A, B$ may be brought respectively to $A^{\prime} B^{\prime}$ (if $A B=A^{\prime} B^{\prime}$ ) by two rotations; the first taking place about a pole situated anywhere in the great circle bending at right angles $A A^{\prime}$, the second about $A^{\prime}$, the position into which it is brought by the first rotation. This view leads us to consider the effect of two rotations taking place successively about two axes fixed in the rotating bolly. Or again, we may make the plane $A^{\prime} B^{\prime}$ revolve into the position $A B$ round a pole

* Communicated by the Author.

$\uparrow$ The italies do not exist in the original. 
taken at the node in which the two planes intersect, and then the points $A, B$ swing into their new positions $A^{\prime}, B^{\prime}$ by means of a rotation about the pole of the great circle, of which $A^{\prime} B^{\prime}$ forms a part. This mode of effecting the displacement naturally suggests the consideration of the effect of rotations taking place successively about two axes fixed in space.

First, then, let us study the effect of the combination of a rotation $(a)$ having $\mathbf{P}$ for its pole, followed by another $(\beta)$, of which $Q$ is the pole, $P$ and $Q$ being points in the surface of the revolving sphere.

In drawing the annexed figure, I have supposed that the two rotations are of the same kind, each tending, when a spectator is standing with his head to the respective poles and his feet to the centre, to make a point to his right-hand pass in front of his face towards his left-hand. Let now $P Q$ revolve through $\frac{a}{2}$ positively into the

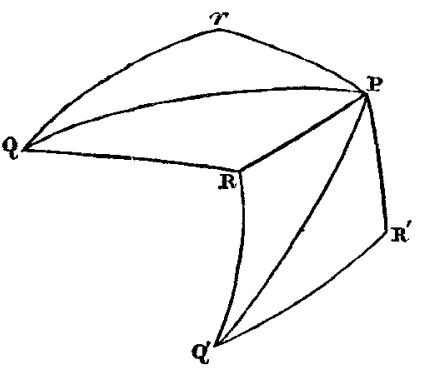
position of $P R$, and through $\frac{\beta}{2}$ negatively into that of $Q R$. Then $I$ say that the two impressed rotations $a$ and $\beta$ about $P$ and $Q$ will be equivalent to a single rotation about $R$, equal to twice the acute angle between $Q R, R P$.

Let the first rotation about $P$ bring $Q$ to $Q^{\prime}$ and $R$ to $R^{\prime}$; it is clear that $Q P R, Q^{\prime} P R, Q^{\prime} P R^{\prime}$ are all equal triangles. Therefore $R^{\prime} Q^{\prime} R=2 P Q R=\beta$. Consequently the positive rotation $\beta$ about $Q^{\prime}$ (the new position of $R$ ) will carry $R^{\prime}$ back again to $R$, its original position. Hence the actual motion which results from the successive rotations combined being consistent with $R$ remaining at rest, must be equivalent to a single rotation about $R$.

To find its magnitude, let the second rotation carry $\mathbf{P}$ to $\mathrm{P}^{\prime *}$; then the angular displacement $\mathrm{PRP}^{\prime}$ (which is the required rotation of the whole body) is equal to twice the acute angle between $Q^{\prime} R, R P$, which is the same as that between $\mathrm{QR}, \mathrm{RP}$, as was to be shown. Thus we see that the semirotations about three poles (considered as the angular points of a spherical triangle), which, taken in order, would bring the sphere back to its first undisturbed position, are equal to the included angles at such poles respectively.

* The reader is requested to fill in the point $P^{\prime}$ and join $P^{\prime} R$. 
If in our figure the order of the rotations had been reversed, $\mathrm{PQ} r, \mathrm{QP} r$ would have been taken respectively equal to $\mathrm{PQR}$, QPR, but in the opposite side of PQ, and $r$ would have been the resultant pole, the resultant rotation remaining in amount the same as before.

If either of the rotations had been negative, the resultant pole would be found in $Q R$ produced, viz. at the intersection of $r \mathrm{Q}$ or $r \mathrm{P}$ with $\mathrm{PQ}$.

Calling the resultant rotation $\gamma$, we have always

$$
\sin \frac{a}{2}: \sin \frac{\beta}{2}: \sin \frac{\gamma}{2}:: \sin \mathrm{QR}: \sin \mathrm{PR}: \sin \mathrm{PQ} \text {. }
$$

When the component rotations are infinitesimal in amount, $\mathrm{R}$ and $r$ will come together in $\mathrm{QP}$; the order of succession of the rotations will be indifferent, and we shall have

$$
\begin{aligned}
a: \beta: \gamma: & : \sin \frac{\alpha}{2}: \sin \frac{\beta}{2}: \sin \frac{\gamma}{2} \\
: & : \sin \mathrm{QR}: \sin \mathrm{RP}: \sin \mathrm{PQ},
\end{aligned}
$$

which gives the rule for the parallelogrammatic composition of two simultaneously impressed rotations*.

If, next, we consider the effect of rotations about two poles, $P$ and $Q$, fixed in space (supposing, as above, that they take place first about $P$ and then about $Q$ ), we must take $P Q r$ equal to half the contrary of the rotation about $\mathrm{P}$, and $\mathrm{PQ} r$ to half the direct rotation about $\mathbf{Q}$ (the angle being now taken positive which was on the first supposition negative, and vice versâ); so that, retaining the original figure, the first rotation will bring $r$ to $\mathrm{R}$, and the second carry $\mathrm{R}$ back to $r$; showing that $r$ is the resultant pole, and that $+\mathrm{P}^{\prime} r \mathrm{P}$, the resultant rotation, will be double the acute angle between $\mathrm{Q} r, r \mathrm{P}$, as in the former case.

To popular apprehension the important doctrine of uniaxial rotation may be made intelligible by the following mode of statement. Take a pocket-globe, open the case and roll about the sphere within it in any manner whatever; then closing the case, there will unavoidably remain two points on the terrestrial surface touching the same two points on the celestial surface as they were in apposition with before the sphere was so turned about in its case.

It is right to bear in mind that the whole of this doctrine is comprised in, and convertible with, the following easy geometrical proposition relative to arcs of great circles on any spherical surface, including the plane as an extreme case.

* Compare Mr. Airy's Tracts, art. "On Precession and Nutation."

$+P^{\prime}$ is not expressed in the figure given. 
"The arcs joining the extremities (each with each in either order) of two other equal arcs, subtend equal angles at either of the points of intersection of two great circles bisecting at right angles the first-named connecting arcs*."

The spherico-triangular mode of compounding rotations given in the above simple disquisition may easily be made the parent of a whole brood of geometrical consequences, which, however, I must leave to the ingenuity and care of those who have a turn for this kind of invention.

But I ought not to omit to invite attention to a remarkable form, which may be imparted to the theorems above stated for the composition of finite rotations, or rather to a theorem which may be derived from them by an obvious process of inference.

Let P, Q, R ...XZ be any number of points on a sphere capable of moving about its centre, joined together by arcs of great circles so as to form a spherical polygon. Imagine any number of rotations to take place about these points in succession as poles. It matters not which is considered the first pole of rotation, but the order of the circulation must be supposed given, as, for instance, P, Q, R ... XZ, or QR...XZP, or R...XZPQ, \&c. This will be one order; the reverse order would be PXZ ... RQ, or QPXZ ... R, \&c.

I shall suppose the circulation to be of the kind first above written. Now we may make two hypotheses:-

1. That the poles are fixed in space.

2. That they are fixed in the rotating body.

In the first case, let the rotations about the given poles $P, Q$, $R, S \ldots X Z$ be double the amounts which would serve to transport $P Q$ to $Q R, Q R$ to $R S$... XZ to $Z P$ respectively.

In the second case, let the rotations be double the amounts which would carry $P Z$ to $Z X \ldots . . S R$ to $R Q, R Q$ to $Q P$ respectively. Then, on either supposition, the sum of the combined rotations is zero; or, to use a more convenient and suggestive form of expression, if the poles of rotation form a closed spherical polygon whose angles are respectively equal to the semirotations about the poles, the resultant rotation is zero.

This proposition is immediately derivable from the fundamental one relative to three poles, given above, by dividing the polygon into triangles by arcs, joining any one of the poles

* This pruposition will be seen to be immediately demonstrable, by the comparison of equal triangles, when viewed as the converse of this other. "' The arcs (or right lines) joining the correspondent extremities of the bases of two similar isosceles spherical (or plane) triangles having a common vertex, are equal to each other." 
with all the rest, or (as pointed out to me by nyy eminent firend Prof. W. Thomson) it becomes apparent as a particular case of a more general proposition, or representing the motion about the successive axes as effected by two equal pyramids having a common vertex at the centre of motion, of which the one is fixed in space, and the other is fixed in the revolving body and rolls over the first, so that the corresponding equal faces are successively brought into coincident apposition.

26 Lincoln's-Inn-Fields,

November 5,1850 .

P.S. To find the pole of rotation whereby PQ may be brought into the position $P^{\prime} Q^{\prime}$, we may use the following simple construction.

Measure off from $O$ the node of the great circles (or right lines) containing $P Q$ and $P^{\prime} Q^{\prime}$, two distances in the proper direction upon each (four distinct assumptions may be made), say $O R$ and $O S$ equal to one another and to the difference between $O P$ and $O P^{\prime}$, then the pole of rotation required, say $\mathrm{E}$, is the centre of the circle described about ROS, and the amount of rotation is the angle subtended by OR or OS at E. The writer of this paper suggests that axis of displacement would be a convenient term for designating the line whereby any finite change in the position of a body moveable about a fixed centre may be brought about; a geometrical theory of rotation leading to the investigation of a very curious species of correlation, now opens upon the view, the general object of which may be stated as follows :-

"Given upon a sphere or plane any curve considered as the locus of successive poles of instantaneous rotation, and the ratio of the rotation abont each pole to its distance from the one that follows*, to construct the curve of the poles of displacement, and to determine the amount of rotation corresponding to each such pole."

The discussion of this question offers a fine field for the exercise of geometrical taste and skill.

LIX. On the Identity of Breislakite and Augite. By Edward J. Chapman, Professor of Mineralogy in University College, London广.

7 THE Breislakite is well known to occur in minute capil1. lary crystals in certain lavas of Vesuvius, and at Capo di Bove near Rome. Its crystalline form has not hitherto * Which by analogy may be termed the "density of rotation."

+ Communicated by the Author. 